\title{
Analysis of cross sections and Vector Analysing Powers of $(d, p)$ reactions within the CDCC+DWBA approach.
}

\author{
P. Chau Huu-Tai ${ }^{1, a}$
}

CEA, DAM, DIF F-91297 Arpajon

\begin{abstract}
Transfer reactions like ${ }^{A} \mathrm{X}(\mathrm{d}, \mathrm{p})^{A+1} \mathrm{X}$ are a powerful tool to investigate nuclear structure properties (see e.g. [1-3]) and the emergence of radioactive beams with high intensity will allow the use of this kind of reactions to study the properties of exotic nuclei. Nevertheless the analysis of reactions involving deuteron remains a difficult task because of its weakly bound and composite features. To overcome these issues, R.C. Johnson et al [4] and G.H. Ratwitcher [5] proposed in the seventies the Continuum Discretized Coupled Channels (CDCC) formalism which explicitly takes into account the composite property of the deuteron and includes the effects on cross sections of the coupling between the breakup and the deuteron ground state channels. Since then the CDCC approach has been widely studied [6-8] and it has been quite successfully applied to analyse elastic [7-9], inelastic [8,10] and transfer cross sections [11-13]. In this contribution, I will propose to re-analyse transfer reactions by the means of CDCC+adiabatic approach for a set of targets with $N=20$ or $N \approx 28$. I will show that with this approach, using both the differential cross sections and the vector analysing powers one can determine the orbital angular momentum and the spin of the captured nucleon without any adjustment.
\end{abstract}

\section{Introduction}

Deuteron induced reactions can be useful to produce radioisotopes of medical interest, to study materials and to investigate nuclei structure especially by means of transfer reactions. These transfer reactions are usually interpreted by using DWBA approach involving global optical potentials for deuteron. In spite of its success to analyse the experimental data, it requires to adjust the optical potential parameters of the entrance channel in order to reproduce the elastic cross sections which means that both the transfer cross sections and the elastic one have been measured. Unfortunately these data are not always available simultaneously. Futhermore this approach does not take into account the deuteron properties (composite weakly bound system with no excited state). We propose here to use the Continuum Discretized Coupled Channels (CDCC) approach and the DWBA approach to interpret the transfer cross sections and the vector analysing power allowing us to get complete information of the captured nucleon without introducing any adjustment even if it is well known that the CDCC description of the elastic cross section remains to be improved at low incident energies. In the second section, I will briefly remind the CDCC and the DWBA formalisms. Then in the third part, I will present some comparisons between the calculations and the experimental data.

\section{CDCC and DWBA approaches}

Proposed independently by R.C. Johnson et al [4] and G.H. Ratwitcher [5], the CDCC formalism assumes that the threebody hamiltonian of the system can be approximated by an effective one and that the deuteron+target system can be described by a simplified wave function which reads as follows:

$$
\begin{aligned}
& \Psi_{J M} \sim \sum_{L=|J-1|}^{J+1}\left[\Phi_{0}(\rho) \otimes \chi_{0}(L, J ; \mathbf{R})\right]_{J M}+ \\
& \sum_{l=0}^{l_{\max }} \sum_{I=|l-S|}^{l+S} \sum_{L=|J-I|}^{J+I} \sum_{i=1}^{N}\left[\tilde{\Phi}_{i}\left({ }^{2 S+1} l_{I} ; \rho\right) \otimes \tilde{\chi}_{i}\left({ }^{2 S+1} l_{I}, L, J ; \mathbf{R}\right)\right]_{J M}
\end{aligned}
$$

where $\Phi_{0}$ and $\tilde{\Phi}_{i}$ denote the deuteron ground state and discretized $p-n$ wave functions, respectively. The main hypotheses in the previous equation is to assume that a truncation in the orbital angular momentum space and a discretization of the p-n continuum into bins can be performed. The correctness of these hypotheses have been cheched by increasing the bin number and by using different ways of discretization. By introducing this trial wave function into the three-body effective Schrödinger equation, one gets a set of coupled channels equations:

$$
\begin{array}{r}
\left(-\frac{\hbar^{2}}{2 \mu_{R}} \frac{d^{2}}{d R^{2}}+\frac{\hbar^{2} L(L+1)}{2 \mu_{R} R^{2}}+V_{p}^{(\text {Coul })}-E_{i}\right) u_{c}^{J}(R)= \\
-\sum_{c^{\prime}} F_{c c^{\prime}}^{J}(R) u_{c^{\prime}}^{J}(R)
\end{array}
$$

This is an Open Access article distributed under the terms of the Creative Commons Attribution-Noncommercial License, which permits unrestricted use, distribution, and reproduction in any noncommercial medium, provided the original work is properly cited. 
where the form factors $F_{c c^{\prime}}^{J}$ are defined by:

$$
F_{c c^{\prime}}^{J}=\left\langle\left[\tilde{\Phi}_{i} \otimes Y_{L}(\hat{R})\right]_{J M}\left|U_{p}+U_{n}\right|\left[\tilde{\Phi}_{i^{\prime}} \otimes Y_{L^{\prime}}(\hat{R})\right]_{J M}\right\rangle_{\hat{R}, \hat{\rho}, \rho}
$$

and are obtained by folding the nucleon-target optical potential by the $p-n$ wave functions. These equations are solved with the following boundary conditions:

$$
u_{c}^{J}(R) \rightarrow \delta_{c_{0} c} U^{(-)}\left(\hat{P}_{i} R\right)-\sqrt{\hat{P}_{i} / \hat{P}_{0}} \hat{S}_{c_{0} c}^{(J)} U^{(+)}\left(\hat{P}_{i} R\right)
$$

to get the S-matrix elements from which the cross sections are calculated.

To deal with transfer reaction, one must go one step further since two partitions $\alpha=A+d$ and $\beta=B+p$ (where $B=A+n$ is the compound nucleus) are now needed to describe the system:

$$
\Psi_{\text {model }}=u_{\alpha}\left(\mathbf{r}_{\alpha}\right) \psi_{\alpha}\left(x_{\alpha}\right)+u_{\beta}\left(\mathbf{r}_{\beta}\right) \psi_{\beta}\left(x_{\beta}\right) .
$$

Assuming that the effect of the coupling of the transfer channels onto the elastic one can be neglected, one can thus write the following set of differential equations:

$$
\left\{\begin{array}{l}
{\left[E_{\alpha}-K_{\alpha}-\left\langle\alpha\left|V_{\alpha \alpha}\right| \alpha\right\rangle\right] u_{\alpha}\left(\mathbf{r}_{\alpha}\right) \approx 0} \\
{\left[E_{\beta}-K_{\beta}-\left\langle\beta\left|V_{\beta \beta}\right| \beta\right\rangle\right] u_{\beta}\left(\mathbf{r}_{\beta}\right)=\int d \mathbf{r}_{\alpha} d \chi_{\beta} \psi_{\beta}^{*} V_{\beta \alpha}\left(\mathbf{r}_{\beta}, \mathbf{r}_{\alpha}\right) \psi_{\alpha} u_{\alpha}\left(\mathbf{r}_{\alpha}\right)}
\end{array}\right.
$$

From this expression it is deduced that the transfer cross section will depend on the properties of the captured nucleon in particular on the transfered orbital angular momentum and on the transfered spin. Therefore some properties of the compound nucleus can be extracted from the transfer cross section and from the vector analysing power.

In the following section, these results will be illustrated for $\mathrm{Ca}, \mathrm{Ti}, \mathrm{Cr}$ and $\mathrm{Fe}$ targets.

\section{Comparison with some experimental data}

In a serie of articles, D.C. Kocher et al [14-16] have measured the transfer cross sections and vector analysing powers for deuteron incident on ${ }^{40} \mathrm{Ca},{ }^{48} \mathrm{Ti},{ }^{52} \mathrm{Cr},{ }^{53} \mathrm{Cr}$, ${ }^{54} \mathrm{Fe}$ and ${ }^{57} \mathrm{Fe}$ targets. For the calcium target the measurements have been performed for energies ranging from 5 to 10 $\mathrm{MeV}$ while for the other targets the incident energy was $10 \mathrm{MeV}$. The transfered orbital angular momentum $l$ can be extracted from the cross sections and from the vector analysing powers (VAP) one can determine whether $j=$ $l+1 / 2$ or $j=l-1 / 2$ as shown by T.J. Yule and W. Haeberli [17]. These data will be used to test whether the previous approach can reproduce the measurements. Some comparisons between the experimental data and the calculations are plotted on figures Fig. 1 and Fig. 2 for ${ }^{40} \mathrm{Ca}$ target, Fig. 3 and Fig. 4 for ${ }^{48}$ Ti target, Fig. 5 and Fig. 6 for ${ }^{52} \mathrm{Cr}$ target, Fig. 7 and Fig. 8 for ${ }^{54} \mathrm{Fe}$ target, Fig. 9 for ${ }^{53} \mathrm{Cr}$ and ${ }^{57} \mathrm{Fe}$ targets and Fig. 10 for ${ }^{57} \mathrm{Fe}$ target. The left part of the figures represent the differential cross sections and the right part the associated VAP. The curves denote the calculations and the dots the experimental data. All the calculations have been performed by using the Koning-Delaroche global optical potential parameterization, the Reid softcore potential to derive the deuteron wave functions ( $S$ and $D$ waves) and by discretizing the continuum into 4 bins for each partial waves. The neutron wave function with given orbital momentum and spin has been obtained with a Woods-Saxon potential whose depth is determined to get the correct excited energy. A good agreement between the experimental data and the calculations has been obtained for the differential cross sections. The agreement is less good for the VAP but the trends are well reproduced allowing a clear assignment of $j$ as illustrated in the lowest part of Fig. 1.

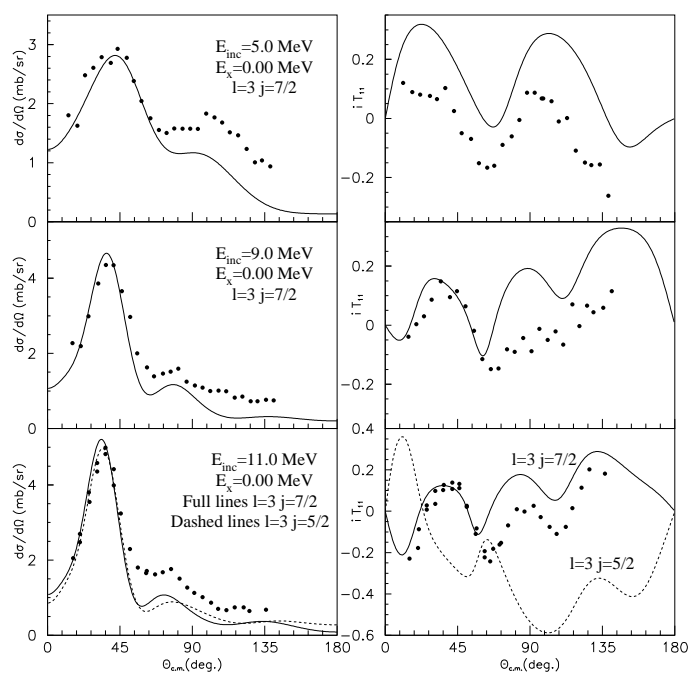

Fig. 1. ${ }^{40} \mathrm{Ca}(\mathrm{d}, \mathrm{p}){ }^{41} \mathrm{Ca}$ cross sections and VAP. The dots represent the experimental data [14]. $E_{i n c}$ and $E_{x}$ denote the incident energy and the energy of the excited state, respectively.

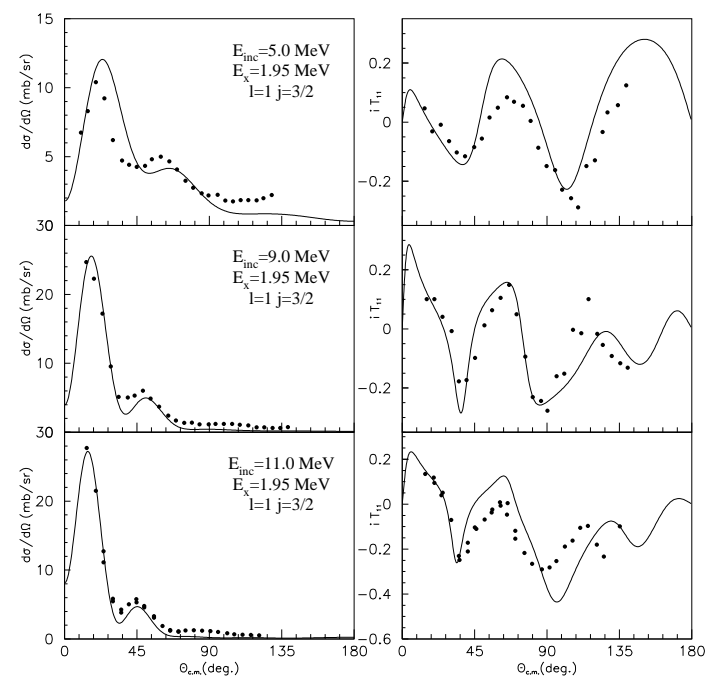

Fig. 2. ${ }^{40} \mathrm{Ca}(\mathrm{d}, \mathrm{p}){ }^{41} \mathrm{Ca}$ cross sections and VAP. The dots represent the experimental data [14]. $E_{i n c}$ and $E_{x}$ denote the incident energy and the energy of the excited state, respectively. 

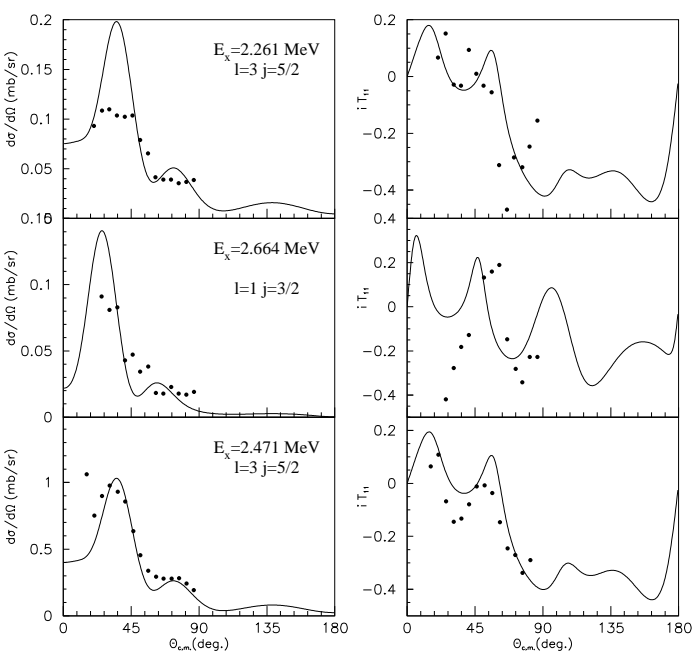

Fig. 3. ${ }^{48} \mathrm{Ti}(\mathrm{d}, \mathrm{p})^{49} \mathrm{Ti}$ cross sections and VAP for deuteron incident at $10 \mathrm{MeV}$. The dots represent the experimental data [15]. $E_{x}$ denotes the energy of the excited state.
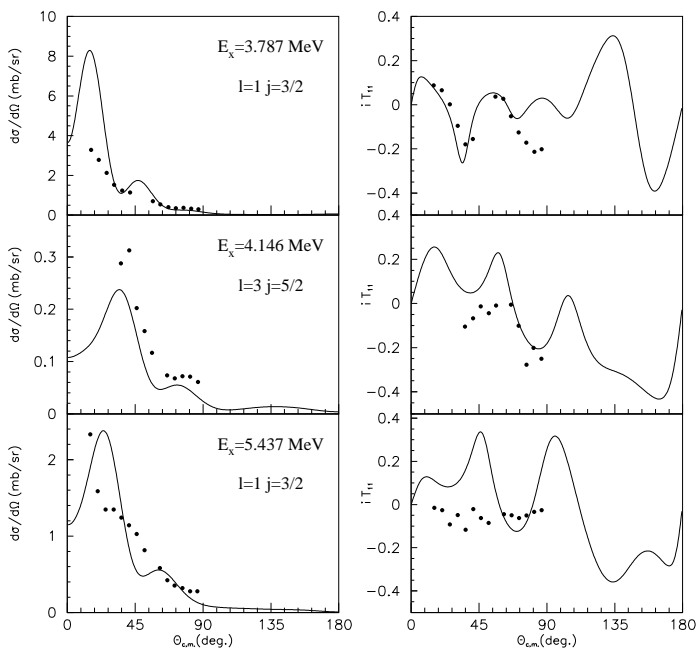

Fig. 4. ${ }^{48} \mathrm{Ti}(\mathrm{d}, \mathrm{p})^{49} \mathrm{Ti}$ cross sections and VAP for deuteron incident at $10 \mathrm{MeV}$. The dots represent the experimental data [15]. $E_{x}$ denotes the energy of the excited state.

\section{Conclusion}

Transfer reactions like (d,p) have been widely used in the past to investigate the properties of nuclei and appear to be a promishing tool to discover the properties of exotic nuclei by means of inverse kinematics and by using high intensity radioactive beams. These reactions have usually been interpreted by using the DWBA approach for which one needs to adjust optical potential parameters for both incoming and outgoing channels. Nevertheless due to the scarceness of experimental data for these exotic nuclei, one can be interested in having an approach as free as possible of any parameter adjustment. The CDCC+DWBA approach owns this property and this contribution like the previous works [11-13] shows its reliability to interpret the
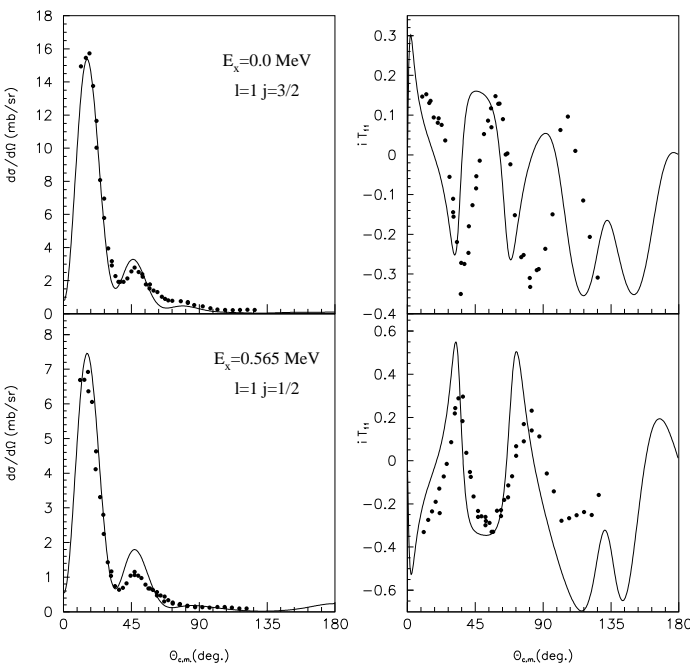

Fig. 5. ${ }^{52} \mathrm{Cr}(\mathrm{d}, \mathrm{p}){ }^{53} \mathrm{Cr}$ cross sections and VAP for deuteron incident at $10 \mathrm{MeV}$. The dots represent the experimental data [15]. $E_{x}$ denotes the energy of the excited state.
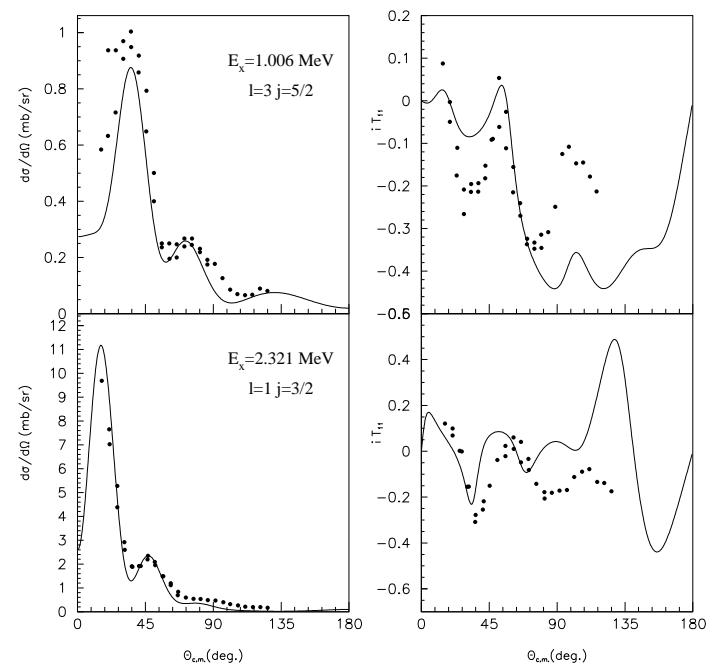

Fig. 6. ${ }^{52} \mathrm{Cr}(\mathrm{d}, \mathrm{p}){ }^{53} \mathrm{Cr}$ cross sections and VAP for deuteron incident at $10 \mathrm{MeV}$. The dots represent the experimental data [15]. $E_{x}$ denotes the energy of the excited state.

experimental data for several even and odd targets even if the elastic cross sections are not always well reproduced.

\section{References}

1. S.T. Butler, Phys. Rev. 80, (1950) 1095.

2. L.D. Knutson and W. Haeberli, Prog. Part. Nucl. Phys. 3, (1980) 127.

3. O. Sorlin and M.-G. Porquet Prog. Part. Nucl. Phys. 61, (2008) 602.

4. R.C. Johnson and P.J.R. Soper, Phys. Rev. C 1, (1970) 976.

5. G.H. Rawitscher, Phys. Rev. C 9, (1974) 2210.

6. N. Austern et al., Phys. Rep. 154, (1987) 125. 


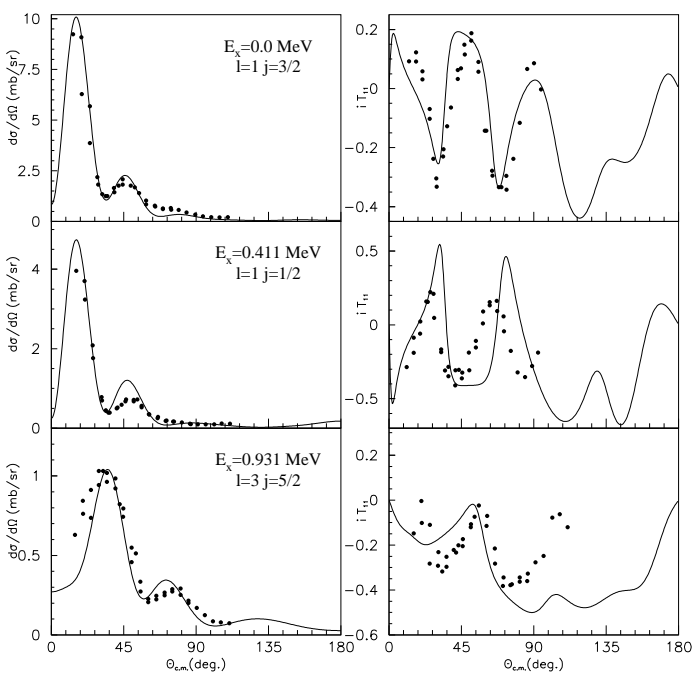

Fig. 7. ${ }^{54} \mathrm{Fe}(\mathrm{d}, \mathrm{p})^{55} \mathrm{Fe}$ cross sections and VAP for deuteron incident at $10 \mathrm{MeV}$. The dots represent the experimental data [15]. $E_{x}$ denotes the energy of the excited state.

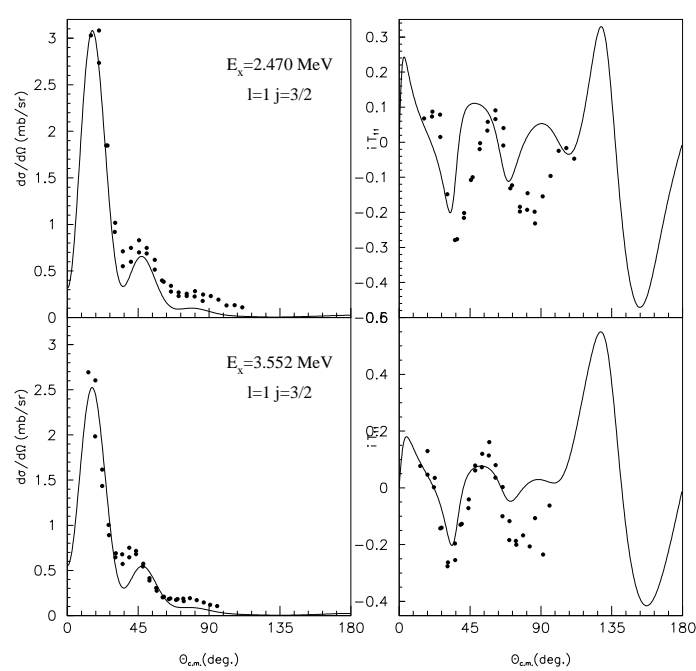

Fig. 8. ${ }^{54} \mathrm{Fe}(\mathrm{d}, \mathrm{p})^{55} \mathrm{Fe}$ cross sections and VAP for deuteron incident at $10 \mathrm{MeV}$. The dots represent the experimental data [15]. $E_{x}$ denotes the energy of the excited state.

7. M. Yahiro and M. Kamimura, Prog. Theor. Phys. 65, (1981) 2046; M. Yahiro and M. Kamimura, Prog. Theor. Phys. 65, (1981) 2051; M. Yahiro et al., Prog. Theor. Phys. 67, (1982) 1467.

8. M. Yahiro et al.,Prog. Theor. Phys. Suppl. 89, (1986) 32.

9. P. Chau Huu-Tai, Nucl. Phys. A773, (2006) 56.

10. P. Chau Huu-Tai, Nucl. Phys. A805, (2008) 483.

11. M. Masaki, Y. Aoki, K. Katoh, S. Nakagawa, N. Nakamoto and Y. Tagishi, Nucl. Phys. A573, (1994) 1.

12. K. Hirota, Y. Aoki, N. Okumura and Y. Tagishi, Nucl. Phys. A628, (1998) 547.

13. M. Iijima, Y. Aoki, A. Ozawa and N. Okumura, Nucl. Phys. A793, (2007) 79.
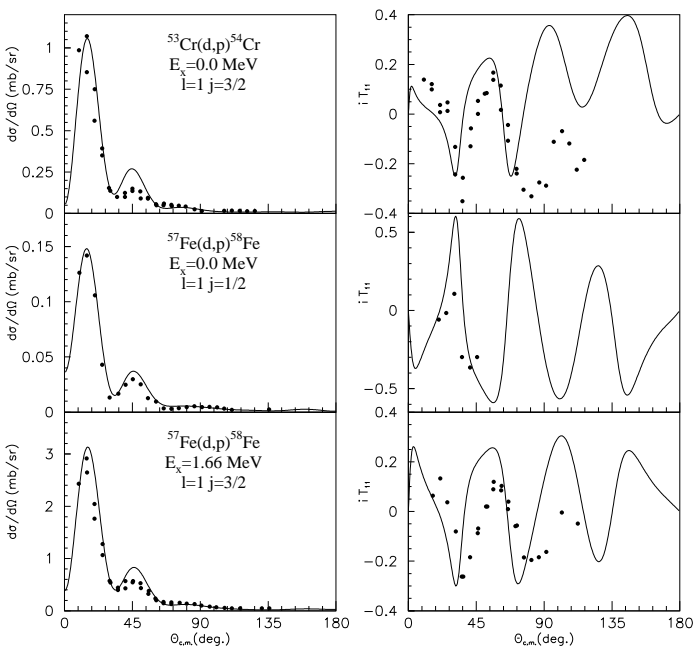

Fig. 9. ${ }^{53} \mathrm{Cr}(\mathrm{d}, \mathrm{p}){ }^{54} \mathrm{Cr}$ and ${ }^{57} \mathrm{Fe}(\mathrm{d}, \mathrm{p}){ }^{58} \mathrm{Fe}$ cross sections and VAP for deuteron incident at $10 \mathrm{MeV}$. The dots represent the experimental data [16]. $E_{x}$ denotes the energy of the excited state.

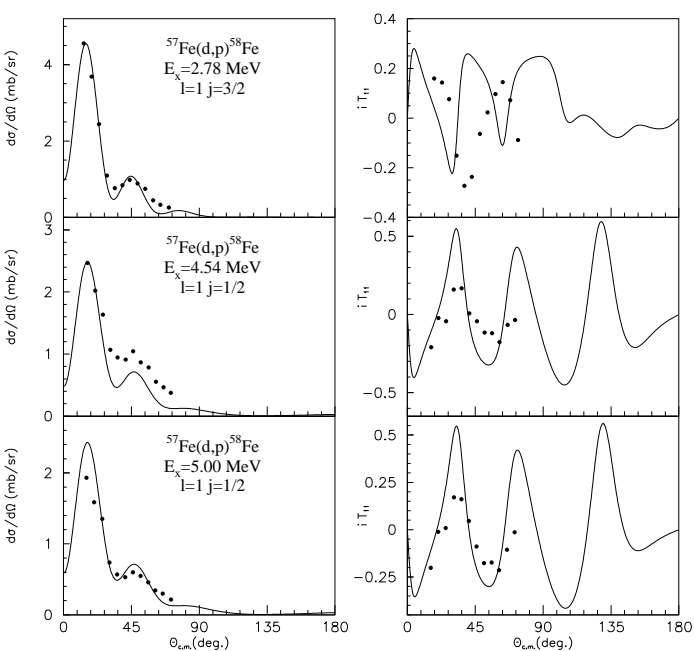

Fig. 10. ${ }^{57} \mathrm{Fe}(\mathrm{d}, \mathrm{p}){ }^{58} \mathrm{Fe}$ cross sections and VAP for deuteron incident at $10 \mathrm{MeV}$. The dots represent the experimental data [16]. $E_{x}$ denotes the energy of the excited state.

14. D.C. Kocher and W. Haeberli, Nucl. Phys. A172, (1971) 652.

15. D.C. Kocher and W. Haeberli, Nucl. Phys. A196, (1972) 225.

16. D.C. Kocher and W. Haeberli, Nucl. Phys. A252, (1975) 381.

17. T.J. Yule and W. Haeberli, Phys. Rev. Lett. 19, (1967) 756; T.J. Yule and W. Haeberli, Nucl. Phys. A117, (1968) 1. 